\title{
Associations between plasma osteopontin levels and the severities of coronary and aortic atherosclerosis
}

\author{
Yukihiko Momiyamaa, ${ }^{a}$, Reiko Ohmori ${ }^{\mathrm{b}}$, Zahi A. Fayad ${ }^{\mathrm{d}}$, Teruyoshi Kiharac, Nobukiyo \\ Tanaka $^{\mathrm{b}}$, Ryuichi Kato ${ }^{\mathrm{b}}$, Hiroaki Taniguchi ${ }^{\mathrm{b}}$, Masayoshi Nagata ${ }^{\mathrm{c}}$, Haruo Nakamura ${ }^{\mathrm{b}}$, and \\ Fumitaka Ohsuzub \\ a Department of Cardiology, National Hospital Organization Tokyo Medical Center, Tokyo, Japan \\ b First Department of Internal Medicine, National Defense Medical College, Saitama, Japan \\ c Iruma Heart Hospital, Saitama, Japan \\ d Mount Sinai School of Medicine, New York, USA
}

\begin{abstract}
High levels of osteopontin (OPN) mRNA and proteins were reported in atherosclerotic plaques [1-3]. OPN-overexpressing transgenic mice developed marked atherosclerotic lesions [4]. These suggest that OPN plays an important role in atherosclerosis. Regarding blood OPN levels, we reported plasma OPN levels to be higher in patients with coronary artery disease (CAD) than without CAD [5]. However, some overlap in OPN levels was found between patients with and without CAD. Since atherosclerotic process is a generalized process that may involve the entire vasculature, we hypothesized that plasma OPN levels may reflect not only coronary atherosclerosis but also atherosclerosis in other vascular beds.
\end{abstract}

Recently, magnetic resonance imaging (MRI) has become a useful tool for non-invasively evaluating atherosclerotic plaques in thoracic and abdominal aortas [6]. Using aortic MRI, we investigated the associations of plasma OPN levels with coronary and aortic atherosclerosis in 136 patients undergoing coronary angiography for suspected or known CAD. Patients with ACS, or aortic or valvular diseases were excluded. Our study was approved by institutional ethics committee. After informed consent was obtained, fasting blood samples were taken, and MRI was performed within 2 weeks of angiography. Plasma OPN levels were measured by ELISA (Human OPN assay kit, IBL).

Aortic MRI was performed on Signa 1.5T Cvi (GE Medical Systems). Transverse proton density-weighted and T2-weighted images of thoracic and abdominal aorta were obtained using double-inversion-recovery FSE sequence: $\mathrm{TR}=2 \mathrm{RR}$ intervals, TE $=10$ (PDW) and $60 \mathrm{~ms}$ (T2W), 20-cm FOV, 4-mm slice thickness, and 8-mm inter-slice gap. As shown in Fig. 1, 9 slices of thoracic aorta and 9 slices of abdominal aorta were obtained at 12-mm intervals. The severity of aortic atherosclerosis was represented as the number of slices with plaque (plaque slice number) and the sum of scores (plaque extent score). On coronary angiograms, the degree of stenosis was evaluated by 5 grades $(\leq 25 \%, 26-50 \%, 51-75 \%$, $76-90 \%,>90 \%$ stenosis). The severity of coronary atherosclerosis was represented as the number of $>50 \%$ stenotic vessels and the numbers of $>50 \%$ and $>25 \%$ stenotic segments. Differences between 2 groups were evaluated by unpaired $t$-test or Chi-square test. Differences among $\geq 3$ groups were evaluated by ANOVA with Scheffe's test. Correlations

(C) 2009 Elsevier Ireland Ltd. All rights reserved.

*Corresponding author. Department of Cardiology, National Hospital Organization Tokyo Medical Center, 2-5-1 Higashigaoka, Meguro-ku, Tokyo 152-8902, Japan Tel.: +81 033411 0111; fax: +81 033412 9811. ymomiyamajp@ yahoo.co.jp (Y. Momiyama). 
between OPN levels and the severities of coronary or aortic atherosclerosis were evaluated by Spearman's correlation test. A $p$ value $<0.05$ was considered statistically significant.

Of the 136 patients, 96 (71\%) had CAD (>50\% stenosis) on angiograms. Thoracic and abdominal aortic plaques were detected by MRI in $88(65 \%)$ and $124(91 \%)$ patients (Table 1). Compared with 40 patients without CAD, 96 with CAD had higher OPN (562 \pm 223 vs. $445 \pm 234 \mathrm{ng} / \mathrm{ml}, p<0.01$ ) and high-sensitivity C-reactive protein (hsCRP)(median 0.78 vs. $0.48 \mathrm{mg} / \mathrm{l}, p<0.02)$ levels. However, OPN levels did not correlate with hsCRP levels. Stepwise increase in OPN levels was found depending on the number of $>50 \%$ stenotic coronary vessels: $445 \pm 234$ in $\mathrm{CAD}(-), 541 \pm 239$ in $1-\mathrm{VD}, 556 \pm 219$ in $2-\mathrm{VD}$, and $604 \pm$ $211 \mathrm{ng} / \mathrm{ml}$ in 3-VD $(p<0.05)$ (Fig. 2). OPN levels correlated weakly with the numbers of $>50 \%$ and $>25 \%$ stenotic segments $(r=0.23$ and $r=0.24, p<0.01)$. Regarding aortic atherosclerosis, plaque slice number and plaque extent score in thoracic aorta correlated with OPN levels $(r=0.23$ and $r=0.23, p<0.01)$. Plaque slice number and plaque extent score in abdominal aorta also correlated with OPN levels $(r=0.18$ and $r=0.22, p<0.05)$. However, total plaque slice number (a total of plaque slice numbers in both aortas) and total plaque extent score (a total of plaque extent scores in aortas) correlated better with OPN levels ( $r=$ 0.26 and $r=0.26, p<0.005$ ). Patients were divided into quartiles by total plaque extent score. OPN levels increased stepwise on quartiles: $451 \pm 165,471 \pm 236,560 \pm 232$, and $629 \pm 252 \mathrm{ng} / \mathrm{ml}(\mathrm{P}<0.02)$ (Fig. 2). In multiple logistic regression analysis, aortic atherosclerosis was the only factor associated with OPN levels independent of atherosclerotic risk factors, but no such significance was found for coronary atherosclerosis. Odds ratio for aortic atherosclerosis (per grade of quartiles) was $1.4(95 \% \mathrm{CI}=1.1-1.9, p<$ 0.01 ) for OPN level $>500 \mathrm{ng} / \mathrm{ml}$.

We previously showed plasma OPN levels to be higher in 107 patients with stable CAD than in 71 without CAD [5]. Although Coskun et al. also reported OPN levels to be higher in 25 patients with stable angina than in 18 without CAD [7], Soejima et al. reported that OPN levels were similar in 60 patients with stable angina and 39 without CAD [8]. Since high OPN mRNA expression was reported in atherosclerotic plaques of aortas [2,3] as well as coronary arteries [1], plasma OPN levels may reflect not only coronary atherosclerosis but also aortic atherosclerosis. OPN was shown to be a constitutive component of elastic fibers of aortas [9]. Serum OPN levels were also high in patients with abdominal aortic aneurysm [10]. In our present study, plasma OPN levels correlated with the severities of both coronary and aortic atherosclerosis. However, in multivariate analysis, only aortic atherosclerosis was an independent factor associated with OPN levels. Hence, OPN levels are more likely to reflect aortic atherosclerosis than coronary atherosclerosis.

Our study has several limitations. First, the number of patients was relatively small. Our study was conducted in Japanese patients undergoing angiography. Our results may not be applicable to general or other ethnic populations. Second, MRI was used to evaluate aortic atherosclerosis, but angiography was used for coronary atherosclerosis. Angiography only shows lumen characteristics. Moreover, because of the small number of study patients, our study could not establish any direct correlation between coronary or aortic atherosclerosis and OPN levels. Third, we did not evaluate ascending aorta or arch to reduce the examination time, because plaques were more prevalent in thoracic descending aorta (45\%) than in ascending aorta (8\%) or arch (31\%) [11]. Finally, most study patients had several atherosclerotic risk factors. Such risk factors could have played a role in high OPN levels in patients with CAD or aortic atherosclerosis. Moreover, $39 \%$ of patients were taking statin. Because OPN levels are affected by statin [12], the inclusion of patients on statin may have confounded our results. 
In conclusion, plasma OPN levels correlated with the severities of both coronary and aortic atherosclerosis. However, OPN levels are more likely to reflect aortic atherosclerosis than coronary atherosclerosis.

\section{References}

1. Giachelli CM, Bae N, Almeida M, et al. Osteopontin is elevated during neointima formation in rat arteries and is a novel component of human atherosclerotic plaques. J Clin Invest. 1993; 92:168696. [PubMed: 8408622]

2. Ikeda T, Shirasawa T, Esaki Y, et al. Osteopontin mRNA is expressed by smooth muscle-derived foam cells in human atherosclerotic lesions of the aorta. J Clin Invest. 1993; 92:2814-20. [PubMed: 8254036]

3. Hirota S, Imakita M, Kohri K, et al. Expression of osteopontin messenger RNA by macrophages in atherosclerotic plaques. Am J Pathol. 1993; 143:1003-8. [PubMed: 8213995]

4. Isoda K, Kamezawa Y, Ayaori M, et al. Osteopontin transgenic mice fed a high-cholesterol diet develop early fatty-streak lesions. Circulation. 2003; 107:679-81. [PubMed: 12578867]

5. Ohmori R, Momiyama Y, Taniguchi H, et al. Plasma osteopontin levels are associated with the presence and extent of coronary artery disease. Atherosclerosis. 2003; 170:333-7. [PubMed: 14612215]

6. Taniguchi H, Momiyama Y, Fayad ZA, et al. In vivo magnetic resonance evaluation of associations between aortic atherosclerosis and both risk factors and coronary artery disease in patients referred for coronary angiography. Am Heart J. 2004; 148:137-43. [PubMed: 15215803]

7. Coskun S, Atalar E, Ozturk E, et al. Plasma osteopontin levels are elevated in non-ST-segment elevation acute coronary syndromes. J Natl Med Assoc. 2006; 98:1746-50. [PubMed: 17128682]

8. Soejima H, Irie A, Fukunaga T, et al. Elevated plasma osteopontin levels were associated with osteopontin expression of CD4+ T cells in patients with unstable angina. Circ J. 2006; 70:851-6. [PubMed: 16799237]

9. Baccarani-Contri M, Taparelli F, Pasquali-Ronchetti I. Osteopontin is a constitutive component of normal elastic fibers in human skin and aorta. Matrix Biol. 1995; 14:553-60. [PubMed: 8535605]

10. Golledge J, Muller J, Shephard N, et al. Association between osteopontin and human abdominal aortic aneurysm. Arterioscler Thromb Vasc Biol. 2007; 27:655-60. [PubMed: 17170369]

11. Agmon Y, Khandheria BK, Meissner I, et al. Relation of coronary artery disease and cerebrovascular disease with atherosclerosis of the thoracic aorta in the general population. Am J Cardiol. 2002; 89:262-7. [PubMed: 11809426]

12. Takemoto M, Kitahara M, Yokote K, et al. NK-104, a 3-hydroxy-4-methilglutaryl coenzyme A reductase inhibitor, reduces osteopontin expression by rat aortic smooth muscle cells. Br J Pharmacol. 2001; 133:83-8. [PubMed: 11325797] 


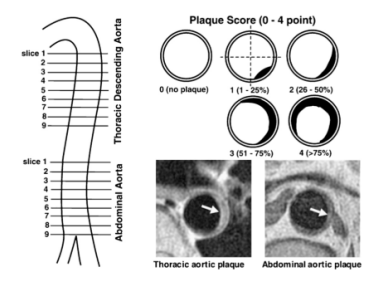

Figure 1.

MRI slices of the aortas and plaque scores. For each patient, 9 slices of thoracic descending aorta and 9 slices of abdominal aorta were obtained at 12-mm intervals, which each covered about $10-\mathrm{cm}$ portion of thoracic aorta below aortic arch and $10-\mathrm{cm}$ portion of abdominal aorta above the bifurcation of iliac artery. Plaque was defined as a clearly identified luminal protrusion with focal wall thickening, and plaque extent in each slice was scored from 0 to 4 points by the percentage of luminal surface involved by plaque. Arrows indicate aortic plaques. 


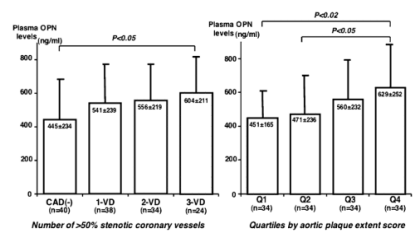

Figure 2.

Associations of plasma OPN levels with the severities of coronary and aortic atherosclerosis. Plasma OPN levels increased stepwise depending on the number of $>50 \%$ stenotic coronary vessels (Left). OPN levels also increased stepwise on quartiles by total aortic plaque extent score (Right). 1-VD, 1-vessel disease; 2-VD, 2-vessel disease; 3-VD, 3-vessel disease. 
Table 1

Clinical characteristics of patients with and without CAD.

\begin{tabular}{lllll}
\hline & All $(\boldsymbol{n}=\mathbf{1 3 6})$ & $\mathbf{C A D}(-)(\boldsymbol{n}=\mathbf{4 0})$ & $\mathbf{C A D}(+)(\boldsymbol{n}=\mathbf{9 6})$ & $(+)$ vs (-) \\
\hline Age (years) & $64 \pm 9$ & $63 \pm 10$ & $64 \pm 9$ & NS \\
Gender (male) & $105(77 \%)$ & $24(60 \%)$ & $81(84 \%)$ & $<0.005$ \\
Hypertension & $85(63 \%)$ & $22(55 \%)$ & $63(66 \%)$ & NS \\
Systolic BP (mmHg) & $132 \pm 19$ & $125 \pm 16$ & $135 \pm 19$ & $<0.005$ \\
Hyperlipidemia & $77(57 \%)$ & $18(45 \%)$ & $59(61 \%)$ & NS \\
Total cholesterol (mg/dl) & $203 \pm 35$ & $205 \pm 30$ & $202 \pm 36$ & NS \\
HDL-cholesterol (mg/dl) & $52 \pm 13$ & $59 \pm 15$ & $49 \pm 12$ & $<0.001$ \\
Statin use & $53(39 \%)$ & $12(30 \%)$ & $41(43 \%)$ & NS \\
Diabetes mellitus & $34(25 \%)$ & $7(18 \%)$ & $27(28 \%)$ & NS \\
Smoking & $55(41 \%)$ & $12(32 \%)$ & $43(45 \%)$ & NS \\
CAD & $96(71 \%)$ & & & $<0.01$ \\
Thoracic aortic plaque & $88(65 \%)$ & $15(38 \%)$ & $73(76 \%)$ & $<0.001$ \\
Abdominal aortic plaque & $124(91 \%)$ & $32(80 \%)$ & $92(96 \%)$ & $<0.01$ \\
Plasma hsCRP (mg/l) (median) & 0.65 & 0.48 & 0.78 & $562 \pm 223$ \\
Plasma OPN (ng/ml) & $528 \pm 232$ & $445 \pm 234$ & & $<0.02$ \\
\hline
\end{tabular}

Data are presented as the mean value $\pm \mathrm{SD}$ or the number $(\%)$ of patients.

Hypertension was defined as blood pressures $\geq 140 / 90 \mathrm{mmHg}$ or on medication.

Hyperlipidemia was defined as total cholesterol levels $>240 \mathrm{mg} / \mathrm{dl}$ or on medication.

Diabetes was defined as fasting glucose levels $\geq 126 \mathrm{mg} / \mathrm{dl}$ or on treatment. 